\title{
Traditional Chinese Medicine Injections in the Treatment of Diabetic Foot: A Systematic Review and Meta-Analysis
}

\author{
Lizi Tan, ${ }^{1}$ Qingyang Shi, ${ }^{1}$ Chunxiang Liu ${ }^{(D)},{ }^{1,2}$ Junhua Zhang $\mathbb{D D}^{1,2}$ \\ Hui Wang $\mathbb{B}^{1,2}$ and Jingbo Zhai ${ }^{1,2}$ \\ ${ }^{1}$ Tianjin University of Traditional Chinese Medicine, Tianjin 300193, China \\ ${ }^{2}$ Evidence-based Medicine Center of Tianjin University of Traditional Chinese Medicine, Tianjin 300193, China
}

Correspondence should be addressed to Chunxiang Liu; liuchunxiangebm@foxmail.com and Junhua Zhang; zjhtcm@foxmail.com Received 30 May 2018; Accepted 23 September 2018; Published 8 October 2018

Guest Editor: Abidemi J. Akindele

Copyright (C) 2018 Lizi Tan et al. This is an open access article distributed under the Creative Commons Attribution License, which permits unrestricted use, distribution, and reproduction in any medium, provided the original work is properly cited.

Context. The role of traditional Chinese medicine injections (TCMIs) in diabetic foot (DF) has not been well estimated. Objective. To evaluate the clinical effective rate, safety, and the financial cost of TCMIs in treating DF and ulcer wound healing. Methods. We searched PubMed, Embase, CENTRAL, China National Knowledge Infrastructure (CNKI), VIP database, and Wanfang database from inception to May 2018 to find all randomized control trials (RCTs) related to TCMIs in DF treatment. The search items were "Traditional Chinese Medicine Injection" AND "Diabetic foot or Diabetic foot ulcer" AND "random". Study Selection and Synthesis. Only RCTs of TCMIs combined conventional therapies versus conventional therapies and that can be quantitatively synthesized were included. Finally, 17 studies and 1294 participants were included after extraction. Two investigators independently extracted and analyzed the data using RevMan5.3 software. Results. The overall clinical effective rate of TCMI groups is higher than that of control groups $[\mathrm{RR}=1.27,95 \mathrm{CI} \%(1.20,1.34), \mathrm{P}<0.00001]$ based on fixed effect model analysis. Regarding motor nerve conduction velocity of median nerve and peroneal nerve, TCMI group showed a significant improvement $(\mathrm{MD}=3.84[2.28,5.41]$, $\mathrm{P}<0.00001 ; \mathrm{MD}=2.89[0.63,5.15], \mathrm{P}=0.01)$. Regarding plasma viscosity TCMI group showed a statistically difference $(\mathrm{MD}=0.27[0.04$, $0.49], \mathrm{P}=0.02)$. In terms of blood viscosity at high shear rate, there was an improvement of TCMI group $(\mathrm{MD}=0.36[0.05,0.67]$, $\mathrm{P}=0.02$ ). However, sensory nerve conduction velocity of peroneal nerve and median nerve showed a contradiction to motor nerve conduction velocity, respectively $(\mathrm{MD}=2.59[-1.69,6.87], \mathrm{p}=0.24 ; \mathrm{MD}=2.73[-0.96,6.43], \mathrm{P}=0.15)$. Conclusion. The data of this study shows that TCMIs can bring benefits to patients with diabetic foot. However, due to low methodological quality of included RCTs, more rigorous designed RCTs with large sample size are recommended to provide more high-quality evidence.

\section{Introduction}

Diabetic foot (DF) is the infection, ulceration, or destruction of tissues of the foot associated with neuropathy and/or peripheral vascular disease (PVD) in the lower extremity of people with diabetes [1]. Diabetic peripheral neuropathy (DPN) and microangiopathy are the most significant risk factors for DF [2]. It is one of the most severe and costly chronic complications of diabetes mellitus (DM) [3]. People with diabetes with foot ulcers experience health expenditures five times higher than those without foot ulcers [4]. It always develops from mild or moderate neural symptoms into diabetic foot ulcers (DFU) on lower extremities even leading to amputations. The amputation rate population with DM is ten to twenty times more than the nondiabetic population [5]. And there is also a twofold risk of mortality for DM population with a history of DFUs compared to those without DM [6].

The prevalence of foot ulcers of people having diabetes mellitus is $4 \%$ to $10 \%$, and the annual population-based incidence is $1.0 \%$ to $4.1 \%[7,8]$. The lifetime incidence of DM people having DFU could be as high as $25 \%$ [9]. Given the rapid growth of DM population which will increase by $48 \%$ in 2045 compared to the number of 425 million [4], we are facing a rapid growing of DF patients in the following 30 years.

However, the DFU is preventable and a timely treatment for ulcers can help in the reduction of severe outcomes. A comprehensive intervention including DFU risk assessments, 
foot care based on prevention, education for patients and their healthcare attendants, and a multidisciplinary treating approach will lower foot complications and amputations by $85 \%$ at most [4].

Being a widely practiced and long-time-used healthcare method, traditional Chinese Medicine plays a significant role in treating DM and glycaemic control [10-15]. According to IDF DIABETES ATLAS 8th edition, intensive glycaemic control is the primary preventive method of DFU and associated with a lower risk of amputation and sensory numbness $[4,16]$. Traditional Chinese medicine injection (TCMI) is sterile liquid of active ingredients that extracted from the natural drugs. The TCMIs with the clinical efficacy of promoting blood circulation to remove blood stasis are now being widely used in China on preventing and treating of DPN and they are subsequent $[17,18]$. However, there was no sufficient evidence-based medicine (EBM) support of that for clinicians and specialists. We performed this systematic review and meta-analysis to investigate the clinical efficacy and safety for TCMIs on DFU.

\section{Methods}

We strictly followed the instruction of Preferred Reporting Items for systematic reviews and meta-analyses: the PRISMA statement during the process of this review [19].

2.1. Inclusion and Exclusion Criteria. We included all the randomized controlled trials (RCTs) applying TCMIs in the treatment of DFU in patients with DM. Participants are diagnosed as diabetic foot and there are no restrictions of age, gender, and course of disease. TCMIs are the injections extracted from herbs, single or mixture herbal formulas. Interventions in trial group are one kind of TCMI with basic care or this TCMI combined conventional therapies with basic care. The basic care and conventional therapies should remain the same in the control group in the same RCT.

Exclusion criteria were as follows: (1) duplicates; (2) systematic reviews and/or meta-analyses; (3) catalogue, indexes, and conferences; (4) irrelevant topics; (5) RCTs using more than one traditional Chinese medicine injection; and (6) studies that cannot be quantitative synthesized.

There were no limits on publication status or language.

2.2. Search Strategy. PubMed, Embase, CENTRAL, China National Knowledge Infrastructure (CNKI), VIP Database for Chinese Technical Periodicals (VIP), and Wanfang databases were searched from inception to May 2018. The search items were "Traditional Chinese Medicine Injection or Zhong Yao Zhu She Ye or Zhu She Ye" AND "Diabetic foot or Diabetic foot ulcer or Tang Niao Bing Zu or Tang Niao Bing Zu Kui Yang” AND "random”.

2.3. Data Extraction. Two reviewers screened and extracted the basic information independently by using a standardized data extraction form of our own and a cross check had been made after the extraction. Disagreements were resolved by discussion and we attempted to contact the authors for the missing data. We used Zotero5.0 software to manage the bibliographies. The information we filled into the form included the following:

(i) General information: title, authors' names, journal, publish date, etc.

(ii) Characteristics of the RCTs: sample size, age, gender, course of disease, interventions, etc.

(iii) Outcomes

(iv) Adverse reactions

\subsection{Types of Outcome Measures}

Primary Outcomes. Clinical effective rates are as follows.

Clinical efficacy was defined as one or more Wagner score reductions after treatment.

Secondary Outcomes. Nerve conduction velocity includes motor nerve conduction velocity $(\mathrm{MCV})$ and sensory nerve conduction velocity (SCV).

Hemorheology includes blood viscosity and plasma viscosity.

2.5. Risk of Bias. Two reviewers made the assessment following the Cochrane Handbook for Systematic Reviews of Interventions 5.1.0 and the systematic review of the methodological quality assessment tools [20, 21].

(i) Random sequence generation (selection bias)

(ii) Allocation concealment (selection bias)

(iii) Blinding of participants and personnel (performance bias)

(iv) Blinding of outcome assessment (detection bias)

(v) Incomplete outcome data (attrition bias)

(vi) Selective reporting (reporting bias)

(vii) Other bias

2.6. Data Synthesis. We conducted this meta-analysis through Revman5.3 software [22]. The categorical variables were analyzed by risk ratio $(\mathrm{RR})$ and the continuous variables take the mean difference (MD) as the effect index, and they are all with $95 \%$ confidence interval (95\% CI).

The heterogeneity among the included studies was analyzed using the chi-square test (the test level was $\alpha=0.1$ ), and the heterogeneity was quantitatively determined using $I^{2}$. If there is no heterogeneity or heterogeneity test result is $\mathrm{P}>0.1$ or $I^{2}<50 \%$, the fixed effect model was applied for metaanalysis. Otherwise, we will further identify the sources of heterogeneity and then reanalyze after reducing the heterogeneity. If there still exists heterogeneity, we will run the analysis with random effects model. Subgroup analyses were conducted based on types of traditional Chinese medicine injections and we illustrated the publication bias of primary outcomes in funnel plot.

\section{Results}

3.1. Study Selection. We finally included 17 studies from 595 studies. The process is demonstrated in Figure 1. 


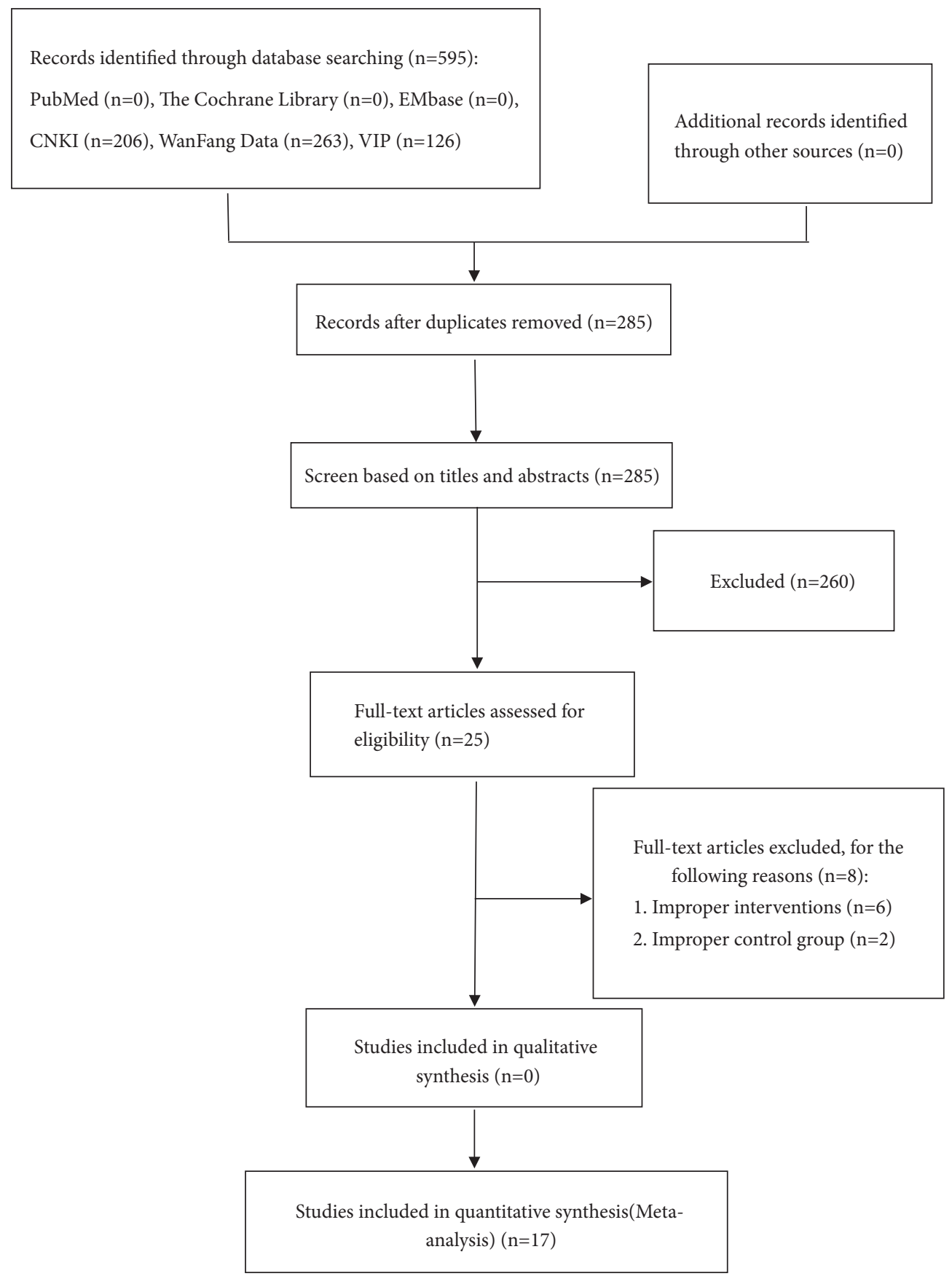

FIGURE 1: Flow diagram of study selection and identification.

3.2. Study Characteristics. A total of 1294 participants were included from the 17 studies [23-39]. All the data were illustrated in Tables 1 and 2 including study size, interventions, and basic information of studies.

All studies utilized Wagner scale for the classification of DFU patients when initially enrolled [40]. Among them, 13 participants were grade $0(1 \%), 232$ were grade $1(17.93 \%), 358$ were grade $2(27.67 \%), 140$ were grade $3(10.82 \%)$, and 41 were grade $4(3.17 \%)$. And $510(39.41 \%)$ were without specific grade classification information.
3.3. Risk of Bias. We used Revman5.3 software to explicitly report the methodological features for each study (Figure 2). Regarding random sequence generation, 14 studies reported "random" without specific method, 2 studies are quasirandomized for their obvious selection bias [27, 36], and only 1 study reported using random number table [34]. Regarding blinding for patients and personnel, 2 studies had high risk and 15 studies had unclear risk. As for blinding for outcome assessment, 13 studies had low risk and 4 studies had unclear risk. Regarding incomplete outcome data, all studies had low 


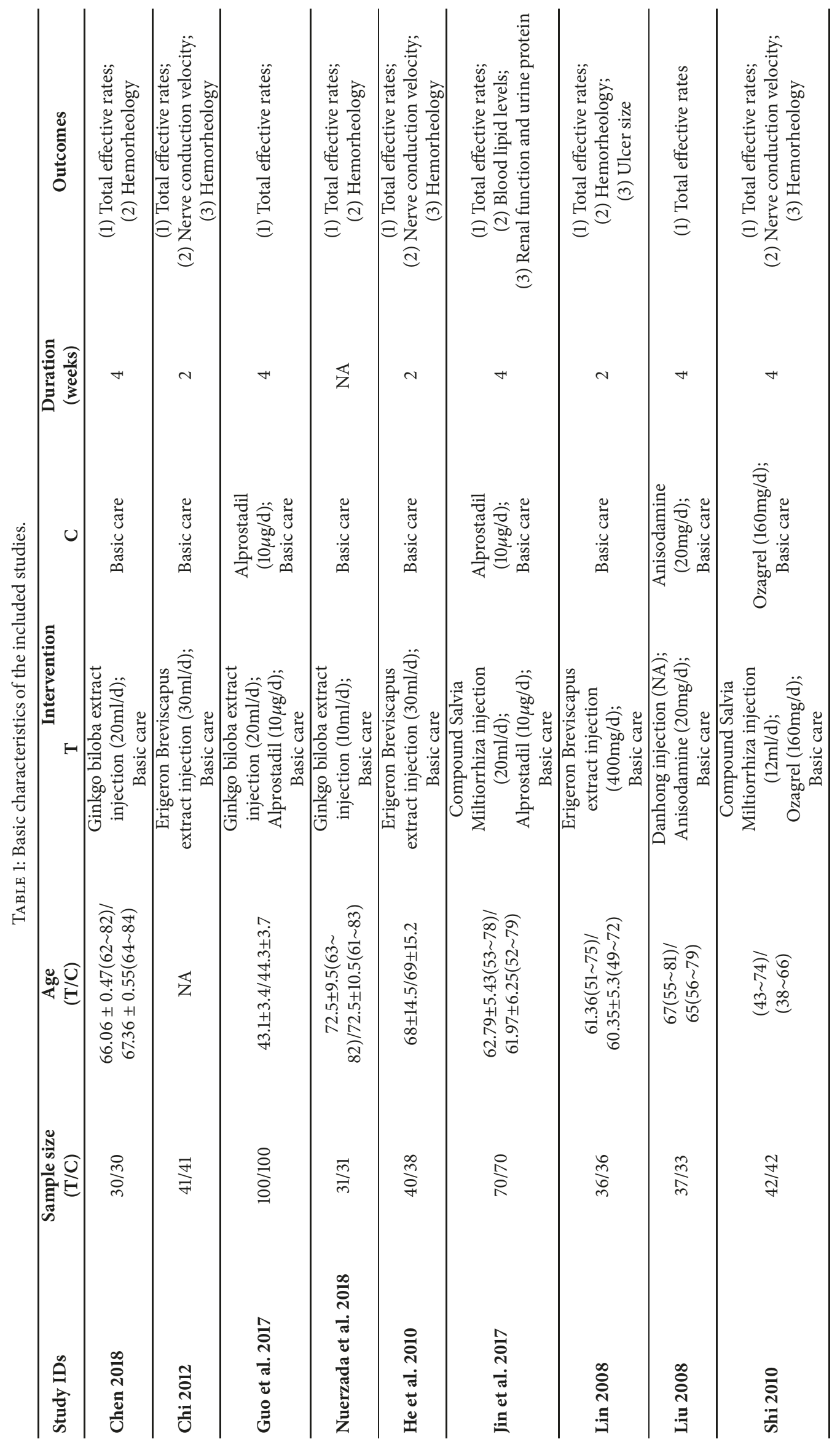




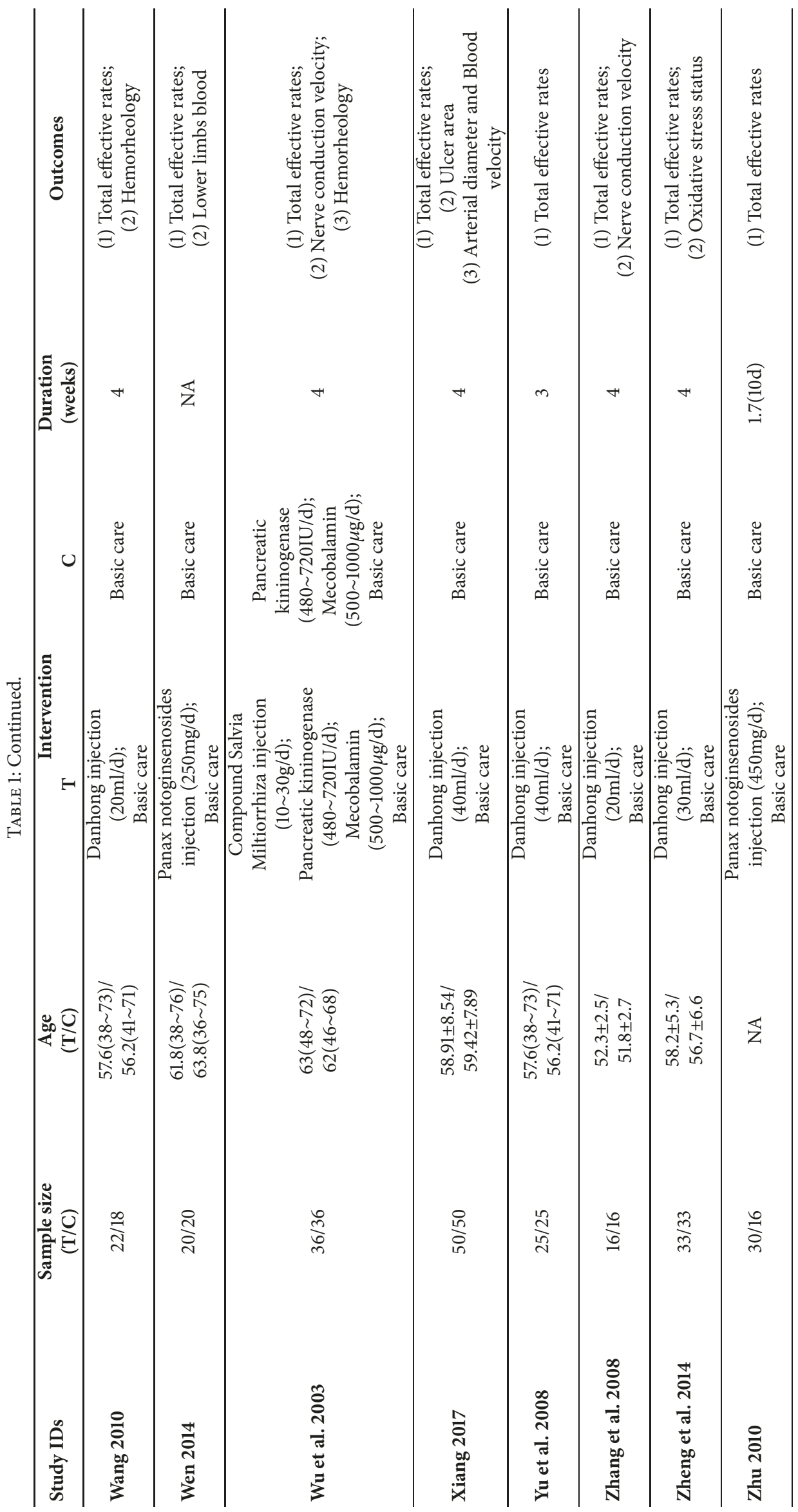




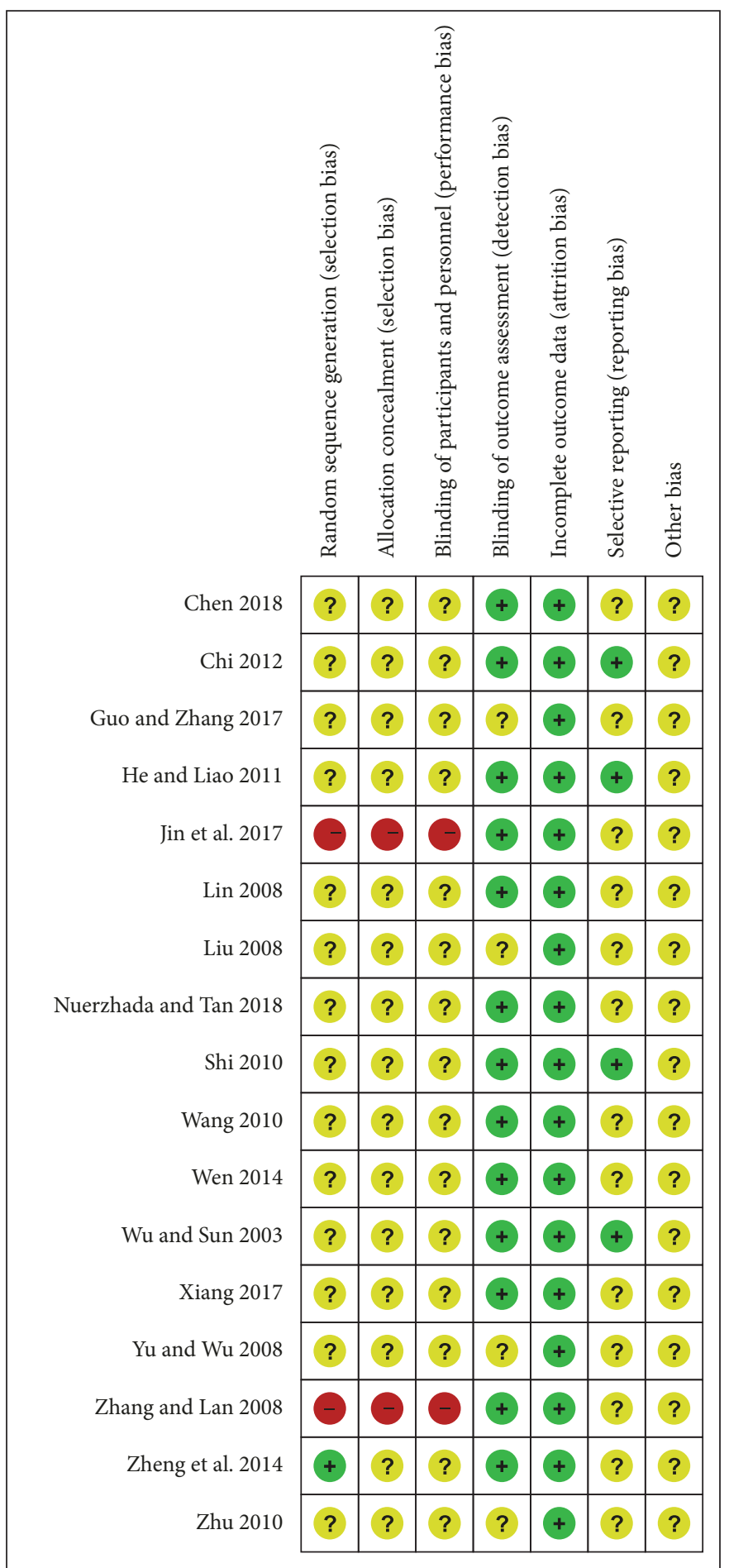

FIGURE 2: Risk of bias summary.

risk of bias. Concerning selective reporting, 4 studies had low risk of bias and 13 studies had unclear risk of bias.

\subsection{Meta-Analysis Results}

3.4.1. Primary Outcome: Clinical Effective Rate. All the 17 studies and 1294 patients receiving treating were included.
The overall clinical effective rate of TCMI groups is higher than that of control groups $(\mathrm{RR}=1.27,95 \mathrm{CI} \%$ [1.20, 1.34], $\mathrm{P}<0.00001)$. Analysis results of different subgroups of conventional therapies based on fixed effect model showed that all TCMI groups outperformed the conventional therapies groups (Danhong injection $\mathrm{RR}=1.24[1.10$, 1.41], $\mathrm{P}=0.0005$; Erigeron Breviscapus extract injection 
TABLE 2: Course of disease.

\begin{tabular}{|c|c|c|c|c|c|}
\hline \multirow{2}{*}{ Study IDs } & \multirow{2}{*}{ Women (\%) } & \multicolumn{2}{|c|}{ Course of Diabetes Mellitus (yrs.) } & \multicolumn{2}{|c|}{ Course of Diabetic Foot } \\
\hline & & Treatment group & Control group & Treatment group & Control group \\
\hline Chen 2018 & $45 \%$ & $9.19 \pm 0.28(5 \sim 15)$ & $9.21 \pm 0.33(6 \sim 15)$ & $38.24 \pm 1.09$ (32 78) days & $37.94 \pm 0.98(30 \sim 76)$ days \\
\hline Chi 2012 & NA & NA & NA & NA & NA \\
\hline Guo 2017 & $41 \%$ & $9.6 \pm 2.9$ & $9.2 \pm 3.5$ & NA & NA \\
\hline Nuerzada 2018 & $47 \%$ & $(5 \sim 14)$ & $(5 \sim 15)$ & NA & NA \\
\hline He 2010 & $46 \%$ & $15 \pm 4.2$ & $16 \pm 4.5$ & $5.7 \pm 1.5$ yrs. & $6.8 \pm 1.8$ yrs. \\
\hline Jin 2017 & $46 \%$ & $15.92 \pm 4.37(8 \sim 20)$ & $16.35 \pm 4.42(8 \sim 21)$ & $1.29 \pm 0.34(0.08 \sim 3) \mathrm{yrs}$. & $1.31 \pm 0.26(0.08 \sim 3)$ yrs. \\
\hline Lin 2008 & $46 \%$ & $11.2(5 \sim 20)$ & $10.56(6 \sim 18)$ & 15.5 (6 66) mos. & $18(4 \sim 72)$ mos. \\
\hline Liu 2008 & $46 \%$ & \multicolumn{2}{|c|}{$11.2(5 \sim 32)$} & NA & NA \\
\hline Shi 2010 & $50 \%$ & $(5 \sim 20)$ & $(2 \sim 25)$ & NA & NA \\
\hline Wang 2010 & $50 \%$ & $11.7(4 \sim 19)$ & $12.4(3 \sim 21)$ & 13 (3 31) mos. & $13.8(2.5 \sim 30)$ mos. \\
\hline Wen 2014 & $22.5 \%$ & $12.4(2 \sim 20)$ & $13.4(3 \sim 21)$ & NA & NA \\
\hline Wu 2003 & $50 \%$ & $7.6(2 \sim 20)$ & $7.8(1.5 \sim 22)$ & NA & NA \\
\hline Xiang 2017 & $42 \%$ & NA & NA & $7.8 \pm 2.3$ mos. & $6.9 \pm 2.4$ mos. \\
\hline Yu 2008 & $40 \%$ & $11.7(4 \sim 19)$ & $12.4(3 \sim 21)$ & 13 (3 31) mos. & $13.8(2.5 \sim 30)$ mos. \\
\hline Zhang 2008 & $40 \%$ & $5.4 \pm 0.3$ & $5.39 \pm 0.27$ & $10.1 \pm 0.8$ mos. & $9.5 \pm 1.0$ mos. \\
\hline Zheng 2014 & $42.4 \%$ & NA & NA & $27.0 \pm 3.9$ mos. & $29.2 \pm 4.0$ mos. \\
\hline Zhu 2010 & $65 \%$ & \multicolumn{2}{|c|}{$(5 \sim 25)$} & NA & NA \\
\hline
\end{tabular}

$\mathrm{RR}=1.39[1.19,1.62], \mathrm{P}<0.0001$; Compound Salvia Miltiorrhiza injection $\mathrm{RR}=1.25[1.12,1.38] \mathrm{P}<0.0001$; Ginkgo Biloba extract injection $\mathrm{RR}=1.17[1.08,1.27] \mathrm{P}=0.0003$; Panax Notoginsenosides injection $\mathrm{RR}=1.69[1.23,2.33] \mathrm{P}=0.001$ ) (Figure 3 ).

3.4.2. Secondary Outcomes. All the data were analyzed on random effect model due to the heterogeneity.

(i) Nerve conduction velocity of median nerve (Figure 4)

MCV: 4 studies and 262 participants were included [23, 27, 28, 31]. TCMI group showed a significant improvement $(\mathrm{MD}=3.84[2.28,5.41], \mathrm{P}<0.00001)$.

SCV: 4 studies and 263 participants were included $[23,27$, $28,31]$. There was no statistical difference between two groups $(\mathrm{MD}=2.59[-1.69,6.87], \mathrm{p}=0.24)$.

(ii) Nerve conduction velocity of peroneal nerve (Figure 5)

MCV: 4 studies and 264 patients were included [23, 27, 28, 32]. TCMI group showed a statistical difference $(\mathrm{MD}=2.89[0.63,5.15], \mathrm{P}=0.01)$.

SCV: 4 studies and 265 patients were included $[23,27,28$, 32]. There was no statistical difference $(\mathrm{MD}=2.73[-0.96,6.43]$, $\mathrm{P}=0.15)$.

(iii) Hemorheology of plasma viscosity (Figure 6)

A total of 5 studies and 256 participants were included [23, 28, $29,31,32]$. It showed a statistical difference $(\mathrm{MD}=0.27[0.04$, 0.49], $\mathrm{P}=0.02$ ).

(iv) Hemorheology of blood viscosity (Figure 7)

High shear rate: 6 studies and 428 participants were included $[23,24,28,29,31,32]$. There was an improvement of TCMI groups $(\mathrm{MD}=0.36[0.05,0.67], \mathrm{P}=0.02)$.
Median shear rate: 4 studies and 268 participants were included [23, 24, 28, 29]. No statistical difference existed $(\mathrm{MD}=-0.02[-0.15,0.12], \mathrm{P}=0.81)$.

Low shear rate: 4 studies and 268 participants were included $[23,24,28,29]$. TCMI groups showed an improvement $(\mathrm{MD}=1.05[0.14,1.96], \mathrm{P}=0.02)$.

3.5. Adverse Events. Only 3 studies reported adverse events. Four patients had facial redness and headache in study of Chi 2012 [32]. Two patients were with rash and pruritus in study of Jin 2017 [36] and two with the same symptom in study of Xiang 2017 [37]. The adverse events were mild and disappeared afterwards, so there was no sample loss. All the other studies reported no adverse events happening.

3.6. Publication Bias. We evaluated the possibility of publication bias by funnel plot of the clinical effective rate (Figure 8). As shown, it was generally symmetrical representing a low risk of publication bias.

\section{Discussions}

4.1. Summary of Main Results. We finally included 17 studies after extraction. The TCMIs they chose to use as the trail interventions concentrating on 5 different kinds are Danhong injection [25-27, 29, 34, 37], Erigeron Breviscapus extract injection (Dengzhanxixin injection) [24, 31, 32], Compound Salvia Miltiorrhiza injection (Fufang Danshen injection) [23, 28, 36], Ginkgo Biloba extract injection (Shuxuening injection) $[35,38,39]$, and Panax Notoginsenosides injection (Xueshuangtong injection) $[30,33]$. And we run the subgroup meta-analysis based on that.

Regarding clinical effective rate, all the five TCMI groups showed an improvement compared to conventional therapies groups no matter if it is the overall rate or subgroup rate, 


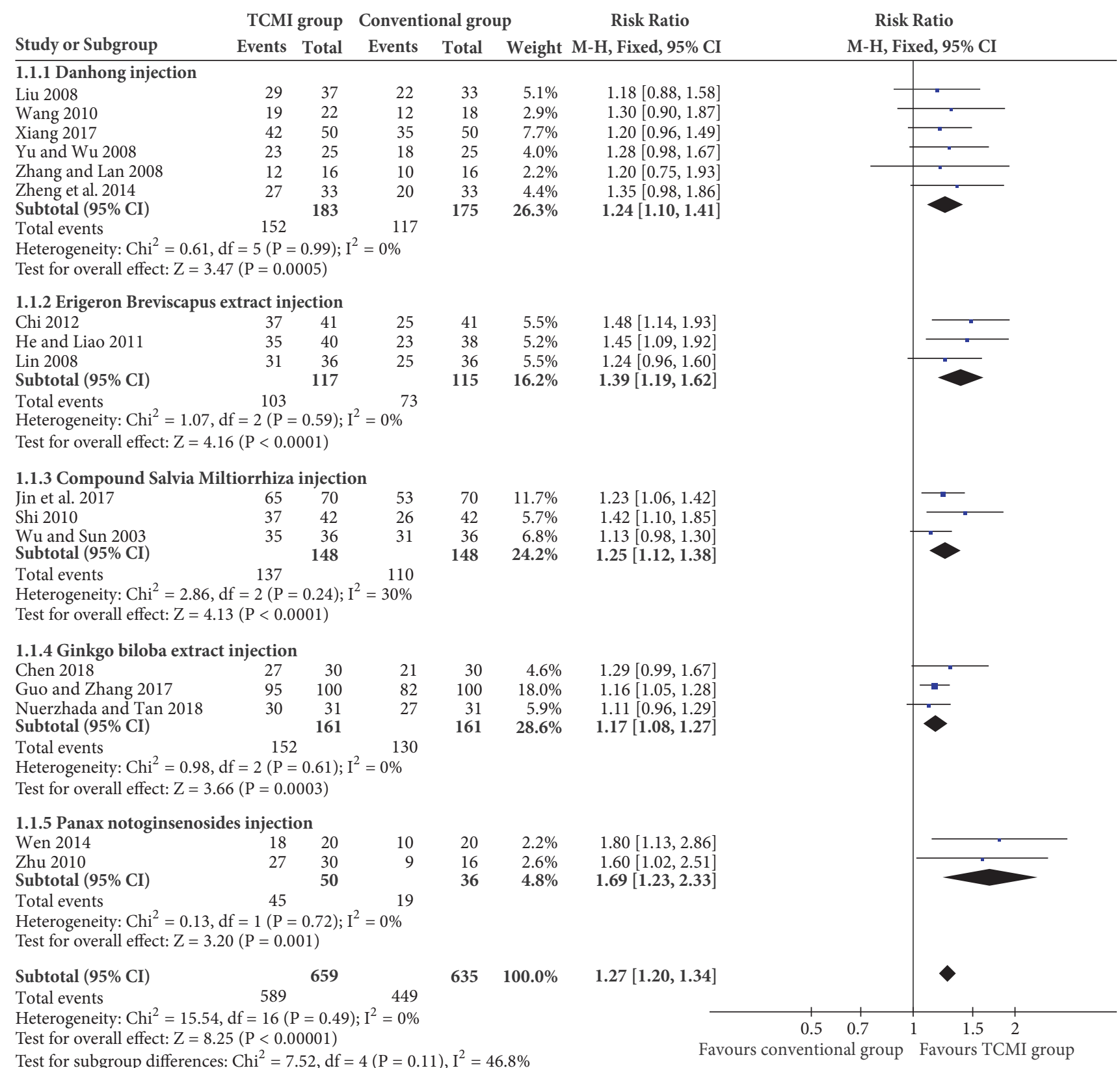

FIGURE 3: Effective rates of TCMI.

respectively.Using TCMIs can significantly raise the rate by $27 \%(\mathrm{P}<0.00001)$ generally. Danhong injection was most widely used in clinic; however, evidence showed that it is not the most effective type to improve the clinical effective rate $(\mathrm{RR}=1.24[1.10,1.41], \mathrm{P}=0.0005)$. Meanwhile, the most effective type, Panax Notoginsenosides injection $(R R=1.69[1.23$, 2.33] $\mathrm{P}=0.001)$, is being used the least. Therefore, more qualified clinical trials and further researches need to be done.

Regarding the secondary outcomes, evidence suggested an improvement of TCMI groups in reducing the plasma viscosity and blood viscosity of high shear rate and low shear rate. And our evidence also supported an improvement of MCV of median and peroneal nerve, whereas no evidence supported the improvement of blood viscosity of median shear rate and SCV of both nerves. Given this contradiction, we consider a further analysis based on more qualified RCTs would help.

4.2. Strength and Limitations. We included 17 studies and 1294 participants totally. No sample loss happened, and all the outcomes were integrally reported at last. Regarding blinding for outcome assessors, 13 studies were evaluated with low risk of publication bias for they measured objective laboratory indexes. Also, test for subgroup difference showed no statistical differences $\left(\mathrm{P}=0.11, I^{2}=46.8 \%\right)$. With no heterogeneity $\left(I^{2}=0 \%, \mathrm{P}=0.49\right)$ in the analysis of overall clinical effective rate and a low heterogeneity (the largest $I^{2}=30 \%, \mathrm{P}=0.24$ ) in subgroups, we considered the internal validity moderate. 


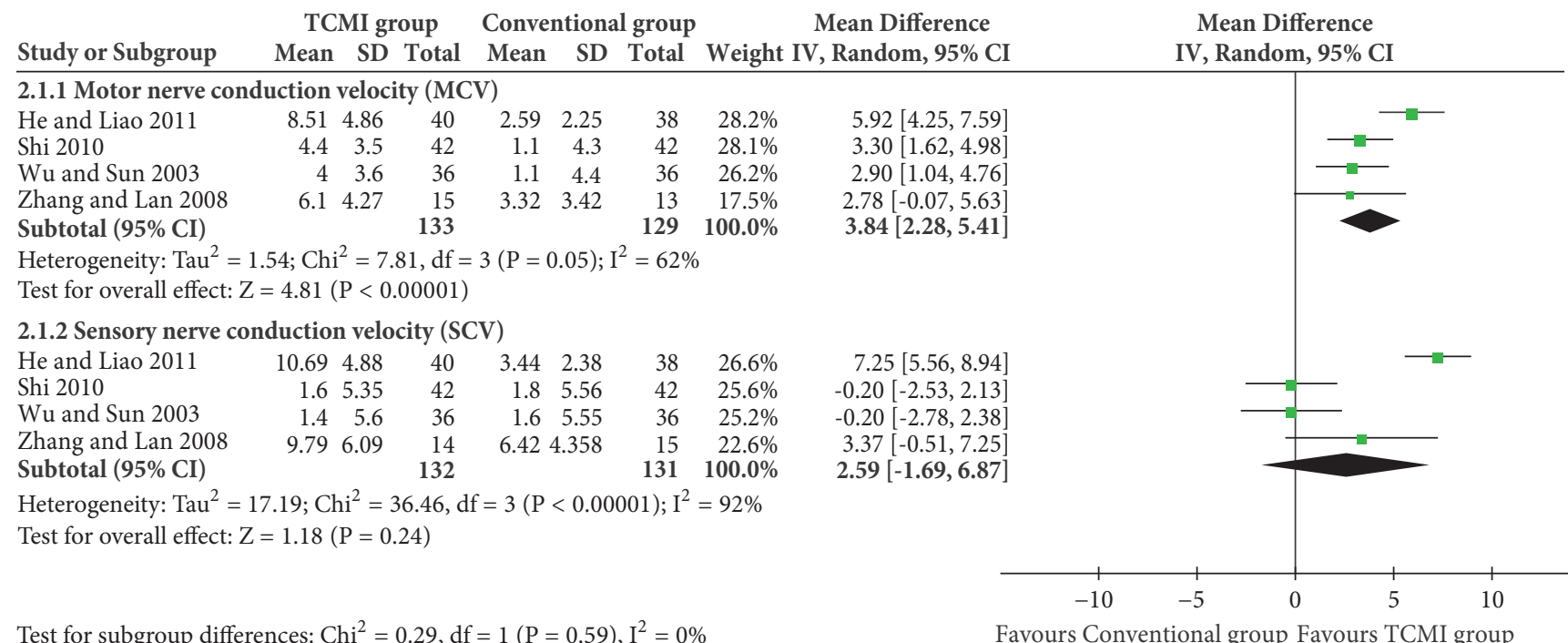

FIgURE 4: Nerve conduction velocity of median nerve.

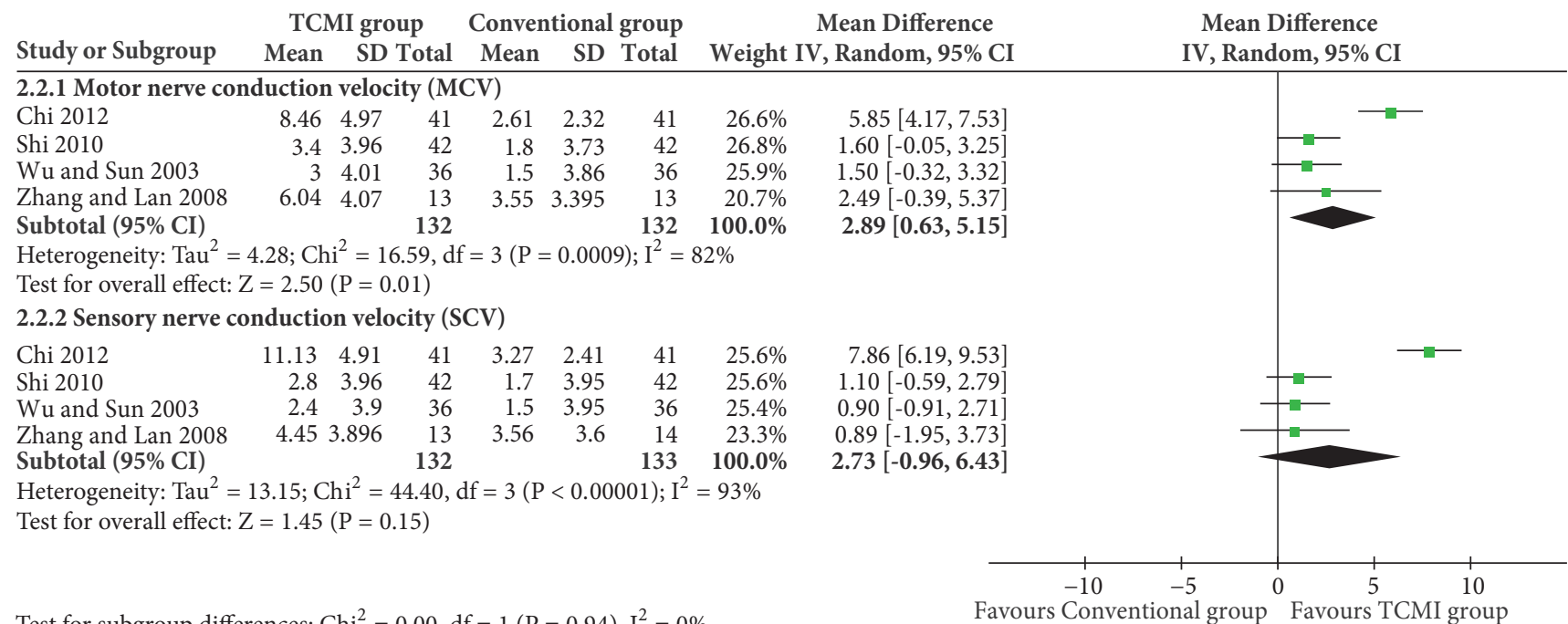

Test for subgroup differences: $\mathrm{Chi}^{2}=0.00, \mathrm{df}=1(\mathrm{P}=0.94), \mathrm{I}^{2}=0 \%$

Favours Conventional group Favours TCMI group

FIGURE 5: Nerve conduction velocity of peroneal nerve.

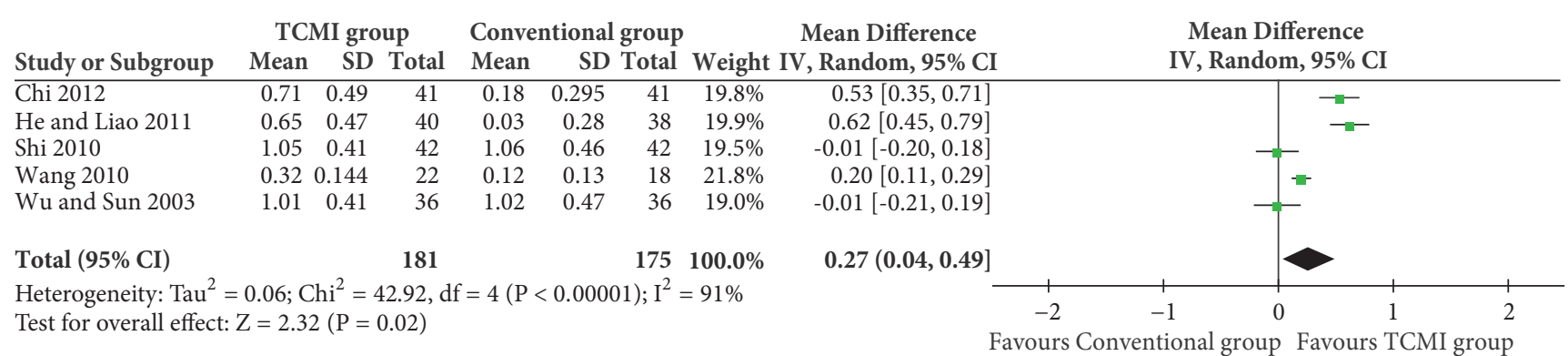

FIGURE 6: Hemorheology of plasma viscosity. 


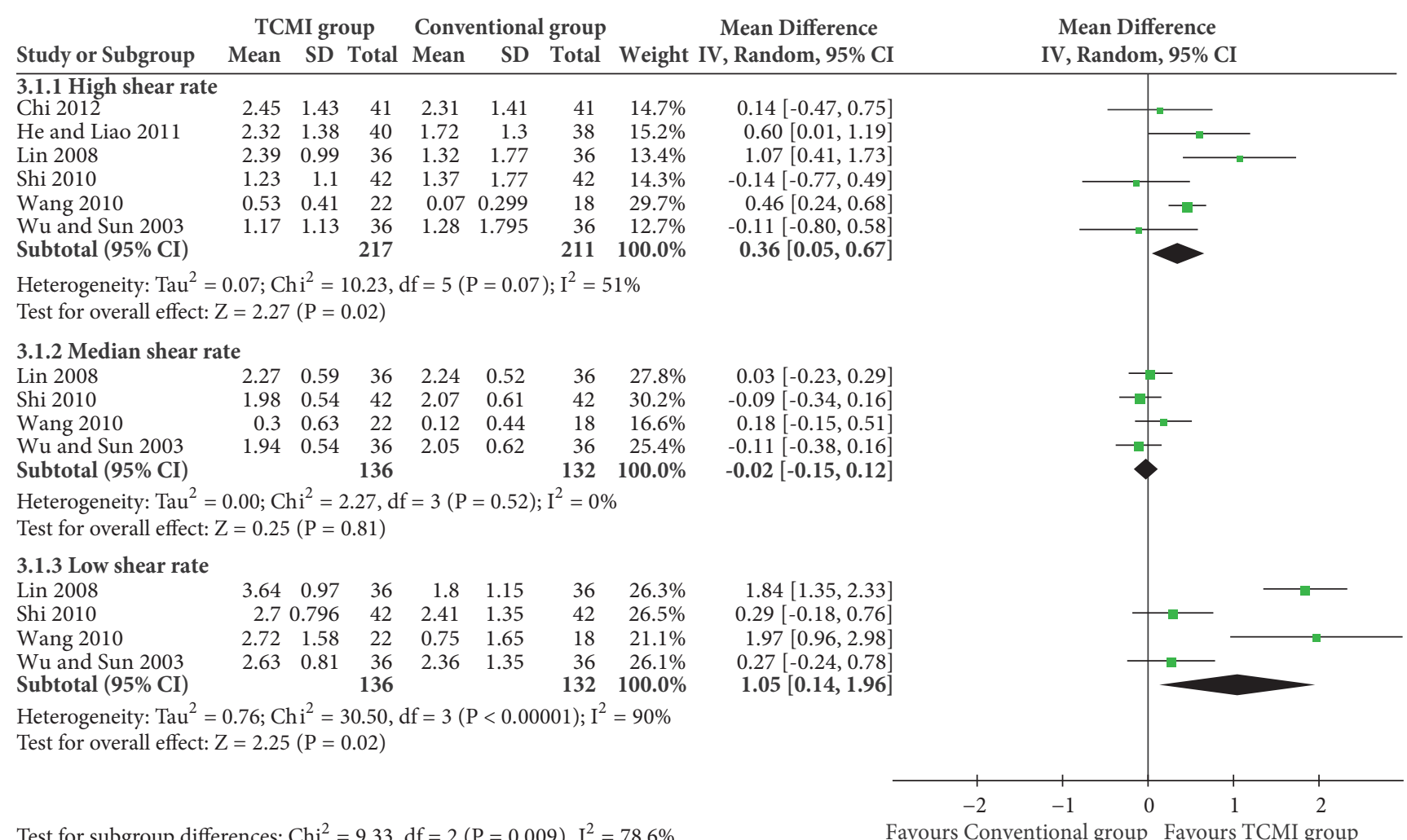

FIGURE 7: Hemorheology of blood viscosity.

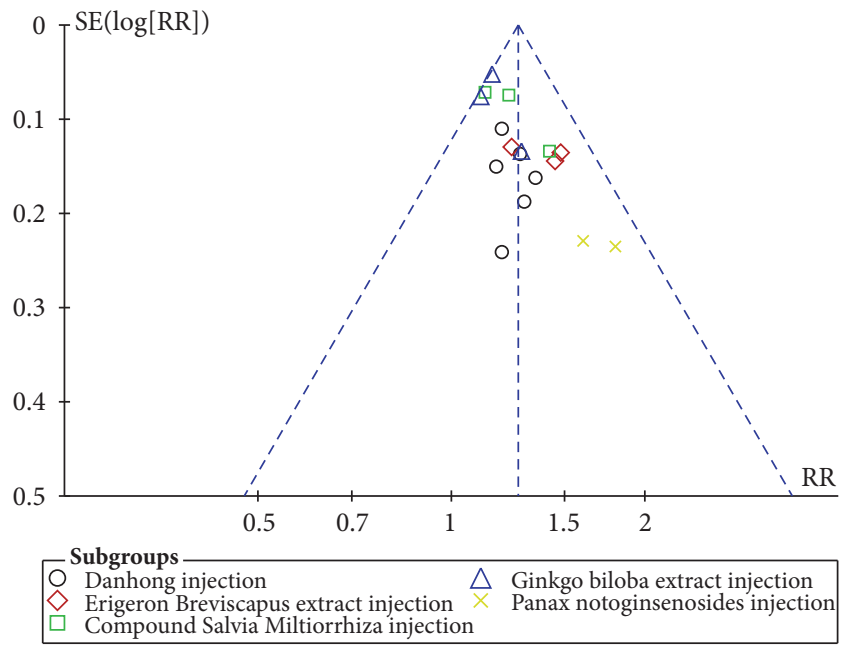

FIgURE 8: Funnel plot of the clinical effective rate.

All participants are enrolled from different regions of mainland China with a balance gender ratio and most of them are middle-aged and elderly people. Within the 17 studies, only 1 reported the random number table and 14 mentioned "random" without the specific approach. Furthermore, 2 are quasi-randomized with an obvious selection bias. None of them mentioned the allocation concealment and the two quasi-randomized trial cannot conceal its allocation.
That indicates a high risk of allocation bias. Besides, no participants included are classified into grade 5 in Wagner scale. Thus, we only recommend the TCMI interventions to clinicians in treating the middle-aged and elderly patients with a mild to moderate DFU classification (with a Wagner scale lower than grade 5).

Few adverse events happened in all the studies and the events happened are mild to moderate degree which will disappear after some resting. And the cost of TCMI is cheap, because most of them are in the Chinese national medical insurance list (Danhong injection, Erigeron Breviscapus extract injection, Ginkgo Biloba extract injection, Panax Notoginsenosides injection) which means $80 \%$ of the expense is covered [41]. According to the course of treatment reported, mostly 28 days, the total cost will be no more than 436.24 CNY. Compared to the significant improvement of clinical effective rate as $27 \%$, TCMIs will only increase the average cost for an ulcer episode by $1.5 \%$ [42]. It can be considered as a cost-effective and safe strategy with a low treatment expense increase.

Although the heterogeneity of primary outcome is low, there may exist potential bias. The courses of disease were inconsistent (Table 2) and the conduction of basic care may differ from practitioners such as debridement and dressing change.

More qualified RCTs need to be included to explain the high heterogeneity in the meta-analysis of secondary outcomes. And due to a contradictory result of secondary outcomes, we hereby recommend more qualified RCTs with 
a report of objective laboratory indexes in treating DFU with TCMIs such as nerve conduction velocity and hemorheology indexes.

\section{Conclusion}

In management of DF, TCMIs can increase the clinical effective rate of conventional therapies by $27 \%$. Along with a better performance in safety and financial burden, the management of DF can be improved by TCMIs. However, the overall methodological and reporting quality of the included studies was limited. Moreover, there are some contradictions in secondary indexes. Therefore, more high-quality large sample-size RCTs are needed to prove and explain it.

\section{Conflicts of Interest}

The authors declare no conflicts of interest regarding the publication of this article.

\section{Acknowledgments}

This work was supported by the Training Program of Innovation Team of Tianjin Higher Education Institution (funding reference no. TD13-5047) through Tianjin Municipal Education Commission. Yu Han in First Teaching Hospital of Tianjin University of Traditional Chinese Medicine provided the information including prices of all the TCMIs.

\section{References}

[1] International Working Group on the Diabetic Foot, "Definitions \& criteria 2015," in Proceedings of the International Working Group on the Diabetic Foot, 2015.

[2] A. Alavi, R. G. Sibbald, D. Mayer et al., "Diabetic foot ulcers," Journal of the American Academy of Dermatology, vol. 70, no. 1, pp. 1.e1-1.e18, 2014.

[3] A. Ibrahim, E. Jude, K. Langton et al., International Diabetes Federation Clinical Practice Recommendations on the Diabetic Foot 2017, International Diabetes Federation, 2017.

[4] Diabetes Atlas, IDF Diabetes Atlas, International Diabetes Federation, 8th edition, 2017.

[5] P. W. Moxey, P. Gogalniceanu, R. J. Hinchliffe et al., "Lower extremity amputations-a review of global variability in incidence," Diabetic Medicine, vol. 28, no. 10, pp. 1144-1153, 2011.

[6] M. M. Iversen, G. S. Tell, T. Riise et al., "History of foot ulcer increases mortality among individuals with diabetes: ten-year follow-up of the Nord-Trøndelag health study, Norway," Diabetes Care, vol. 32, no. 12, pp. 2193-2199, 2009.

[7] International Working Group on the Diabetic Foot, "Epidemiology of diabetic foot infections in a populationbased cohort," in International Consensus on the Diabetic Foot, Noordwijkerhout, the Netherlands, 2003.

[8] L. A. Lavery, D. G. Armstrong, R. P. Wunderlich, J. Tredwell, and A. J. M. Boulton, "Diabetic foot syndrome: evaluating the prevalence and incidence of foot pathology in Mexican Americans and non-Hispanic whites from a diabetes disease management cohort," Diabetes Care, vol. 26, no. 5, pp. 1435-1438, 2003.

[9] N. Singh, D. G. Armstrong, and B. A. Lipsky, "Preventing foot ulcers in patients with diabetes," The Journal of the American Medical Association, vol. 293, no. 2, pp. 217-228, 2005.
[10] X.-L. Tong, L. Dong, L. Chen, and Z. Zhen, "Treatment of diabetes using traditional Chinese medicine: past, present and future," American Journal of Chinese Medicine, vol. 40, no. 5, pp. 877-886, 2012.

[11] F. Lian, G. Li, and X. Chen, "Chinese herbal medicine Tianqi reduces progression from impaired glucose tolerance to diabetes: a double-blind, randomized, placebo-controlled, multicenter trial," The Journal of Clinical Endocrinology \& Metabolism, vol. 99, pp. 648-655, 2014.

[12] H. Cao, M. Ren, L. Guo et al., "JinQi-Jiangtang tablet, a Chinese patent medicine, for pre-diabetes: a randomized controlled trial," Trials, vol. 11, no. 1, 2010.

[13] X. L. Tong, S. T. Wu, F. M. Lian et al., "The safety and effectiveness of TM81, a Chinese herbal medicine, in the treatment of type 2 diabetes: a randomized double-blind placebo-controlled trial," Diabetes, Obesity and Metabolism, vol. 15, no. 5, pp. 448454, 2013.

[14] L. Ji, X. Tong, H. Wang et al., "Efficacy and safety of traditional Chinese medicine for diabetes: a double-blind, randomised, controlled trial," PLoS ONE, vol. 8, no. 2, Article ID e56703, 2013.

[15] F. Lian, J. Tian, X. Chen et al., “The efficacy and safety of Chinese herbal medicine Jinlida as add-on medication in type 2 diabetes patients ineffectively managed by metformin monotherapy: a double-blind, randomized, placebo-controlled, multicenter trial," PLoS ONE, vol. 10, no. 6, Article ID e0130550, 2015.

[16] R. Hasan, B. Firwana, T. Elraiyah et al., "A systematic review and meta-analysis of glycemic control for the prevention of diabetic foot syndrome," Journal of Vascular Surgery, vol. 63, no. 2, pp. 22S-28S.e2, 2016.

[17] C. Zhang, Y.-X. Ma, and Y. Yan, "Clinical effects of acupuncture for diabetic peripheral neuropathy," Journal of Traditional Chinese Medicine, vol. 30, no. 1, pp. 13-14, 2010.

[18] M. Jiyang, D. Gyan, L. Yang, G. Shuang, and J. Xi-cheng, "Clinical application status on anti-inflammatory of traditional classical formula-zhibaidihuang pill," Modern Chinese Medicine, vol. 20, no. 1, pp. 6-13, 2018.

[19] D. Moher, A. Liberati, J. Tetzlaff, and D. G. Altman, "Preferred reporting items for systematic reviews and meta-analyses: the PRISMA statement," British Medical Journal, vol. 339, article b2535, 2009.

[20] X. Zeng, Y. Zhang, J. S. Kwong et al., “The methodological quality assessment tools for preclinical and clinical studies, systematic review and meta-analysis, and clinical practice guideline: a systematic review," Journal of Evidence-Based Medicine, vol. 8, no. 1, pp. 2-10, 2015.

[21] J. Higgins and S. E. Green, "Cochrane Handbook for Systematic Reviews of Interventions Version 5.1.0," in The Cochrane Collaboration, 2011, March 2011, http://www.cochrane-handbook.org.

[22] The Nordice Cochrane Centre and The Cochrane Collaboration, Review Manager (RevMan), Copenhagen, Denmark, 2014.

[23] W. Han-ni and S. Hui, "Study on clinical therapeutic effect of composite salvia injection matched with western medicine in treating diabetic foot," Chinese Journal of Integrative Medicine, vol. 9, no. 4, pp. 312-312, 2003.

[24] Z. Lin, "Clinical Observation on 36 Cases of Diabetic Foot Treated with Conventional Therapy and Erigeron Breviscapus Injection," Journal of New Chinese Medicine, vol. 40, no. 08, pp. 25-26, 2008.

[25] C. Liu, "Clinical Observation of Diabetic Foot Treated with Integrated Chinese Traditional and Western Medicine," Shanxi Journal of Traditional Chinese Medicine, vol. 24, no. 9, p. 32, 2008. 
[26] X. Yu and J. Wu, "Treatment of 25 Cases of Diabetic Foot with Danhong Injection," Zhejiang Journal of Traditional Chinese Medicine, vol. 43, no. 5, p. 303, 2008.

[27] Y. Zhang and Y. Yan, "Clinical Observation of Diabetic Foot Treated by Danhong Injection plus Low Dose PGE1," Diabetology, vol. 4, no. 5, pp. 41-42, 2008.

[28] S. Shi, "Clinical Observation of Diabetic Foot Treated by Compound Salvia Miltiorrhiza Injection Combined with Ozagrel," vol. 19, pp. 738-741, 2010.

[29] G. Wang, "Clinical Observation of 40 Cases of Diabetic Foot Treated by Integrated Chinese Traditional and Western Medicine," Journal of Liaoning University of TCM, vol. 12, no. 11, pp. 177-178, 2010.

[30] S. Zhu, "Clinical Study on Treatment of Diabetic Foot with Xueshuantong," Chinese Journal of Modern Drug Application, vol. 4, no. 15, pp. 105-106, 2010.

[31] J. He and X. Liao, "Efficacy of Erigeron Breviscapus Injection in Treating Diabetic Foot," Guangdong Medical Journal, vol. 32, no. 19, pp. 2599-2600, 2011.

[32] R. Chi, "Clinical Analysis of Diabetic Foot Treated with Erigeron Breviscapus," Hunyuyujiankang Shiyongzhenliao, vol. 12, pp. 17-23, 2012.

[33] R. Wen, "Clinical Observation on Treatment of Diabetic Foot with Xueshuantong Injection," Healthy People, vol. 8, no. 8, pp. 531-532, 2014.

[34] R. Zheng, G. Sun, and C. Chen, "Clinical Observation of Danhong Injection in the Treatment of Diabetic Foot," Chinese Journal of Pharmacoepidemiology, vol. 23, no. 4, pp. 11-13, 2014.

[35] W. Guo and S. Zhang, "To investigate the clinical efficacy of alprostadil combined with Shuxuening in the treatment of early diabetic foot," China Reflexolocy, vol. no. 16, pp. 84-86, 2017.

[36] B. Jin, Y. Cai, and J. Wang, "Study of therapeutic effects of Alprostadil Injection and Composite Salvia Injection on diabetic foot," Drug Evaluation Research, vol. 40, no. 11, pp. 1638-1641, 2017.

[37] S. Xiang, "Fifty Cases of Diabetic Foot Treated with Danhong Injection," Henan Journal of Traditional Chinese Medicine and Pharmacy, vol. 37, no. 4, pp. 661-663, 2017.

[38] D. Chen, "Clinical Observation on Treatment of Diabetic Foot with Shuxuening Injection Combined with Basic Therapy," Journal of Clinical Medical, vol. 5, no. 7, pp. 41-42, 2018.

[39] H. Nuerzada and J. Tan, "Clinical Observation of Shuxuening Injection Combined with Basic Therapy in Treating Diabetic Foot," Clinical Observation of Shuxuening Injection Combined with Basic Therapy in Treating Diabetic Foot, 2018.

[40] F. W. Wagner, "A classification and treatment program for diabetic, neuropathic, and dysvascular foot problems," Instr Course Lect, vol. 28, pp. 143-165, 1979.

[41] Ministry of Human Resources and Social Security of the People's Republic of China, The Ministry of Human Resources and Social Security publishes the national basic medical insurance, industrial injury insurance and maternity insurance drug catalog, Ministry of Human Resources and Social Security of the People's Republic of China, Ministry of Human Resources and Social Security of the People's Republic of China web site, 2017.

[42] V. R. Driver, M. Fabbi, L. A. Lavery, and G. Gibbons, "The costs of diabetic foot: The economic case for the limb salvage team," Journal of Vascular Surgery, vol. 52, no. 3, 2010. 


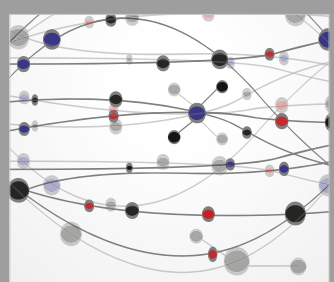

The Scientific World Journal
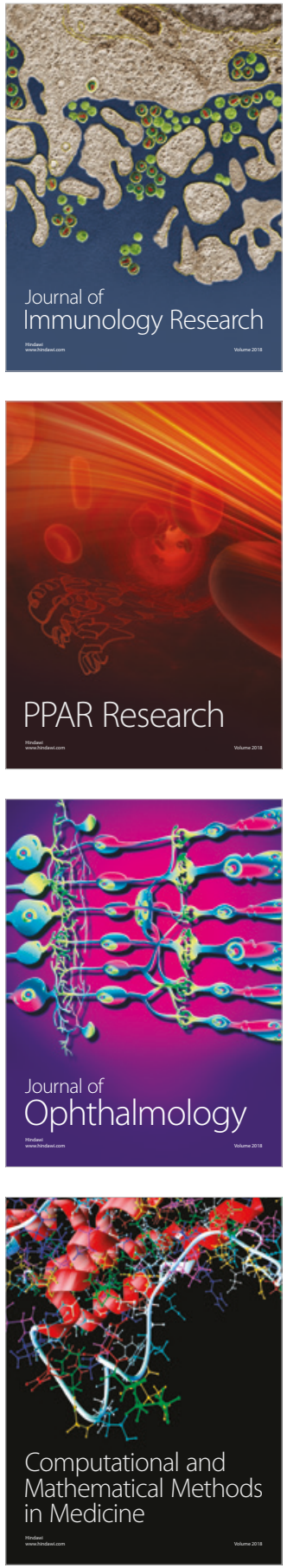

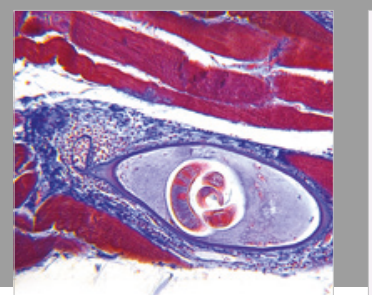

Gastroenterology Research and Practice

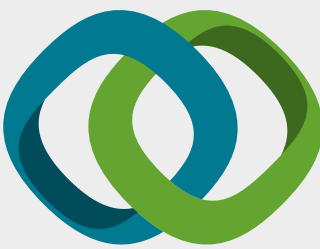

\section{Hindawi}

Submit your manuscripts at

www.hindawi.com
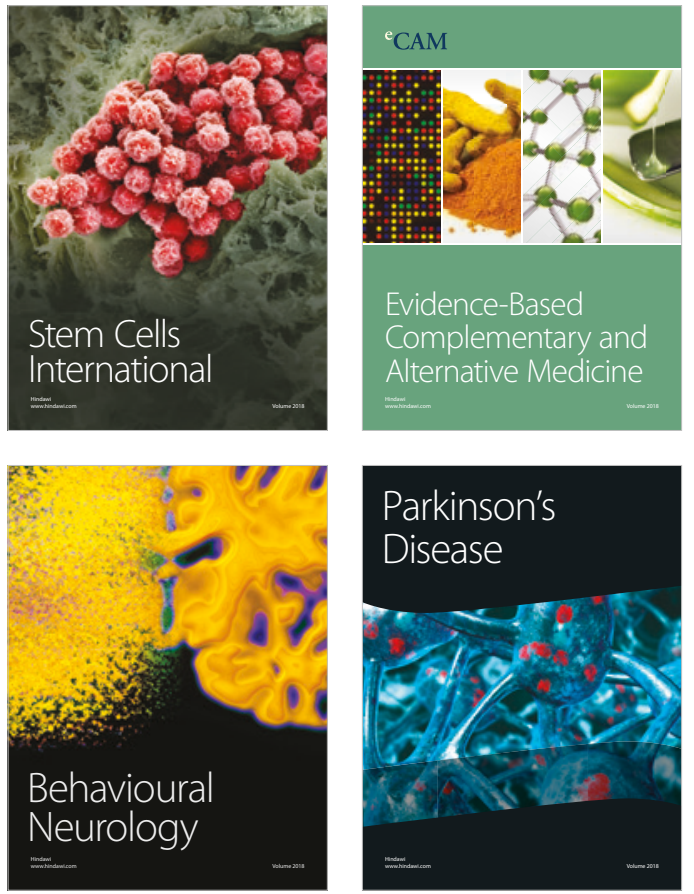

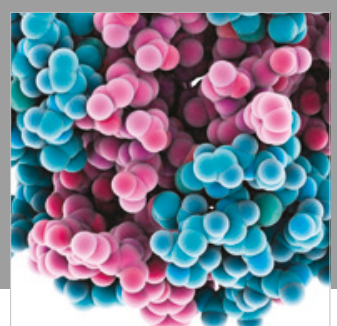

ournal of

Diabetes Research

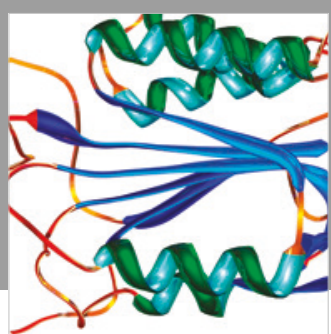

Disease Markers
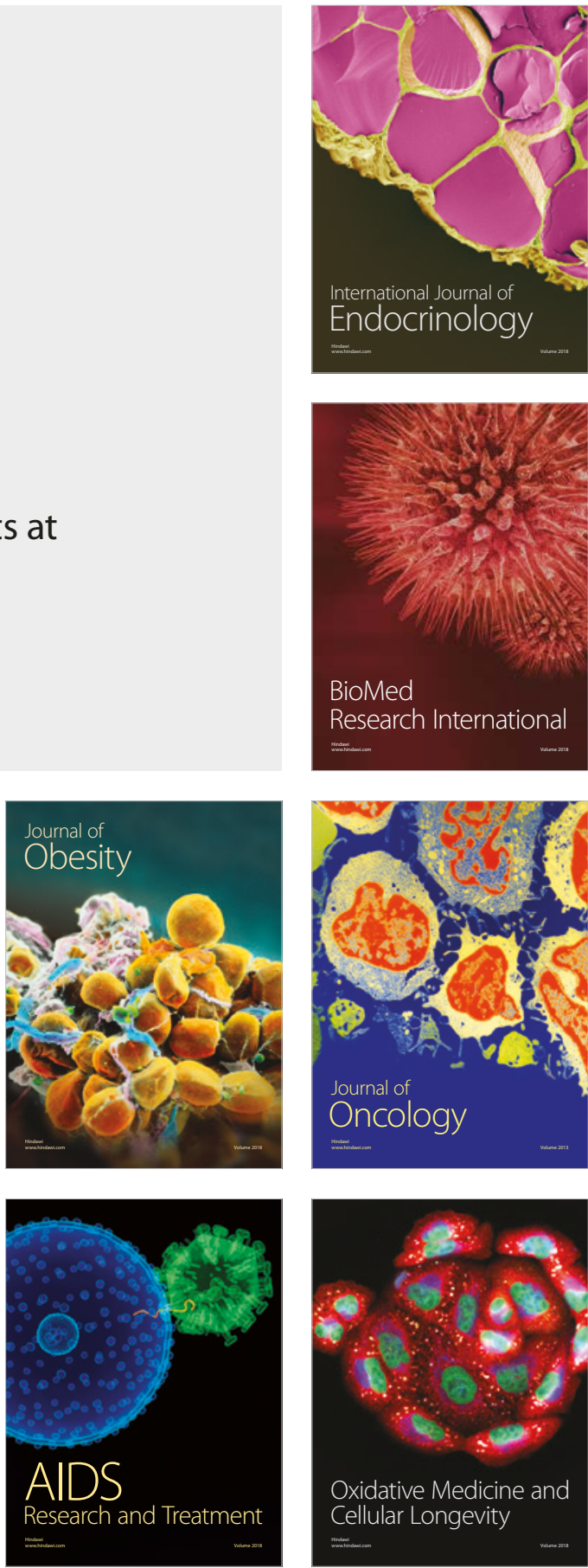